\title{
Does the Phenomenological Approach Contradict the Quantum Theory of Exciton-Polariton Spatial Dispersion?
}

Qi Guo and Sien Chi

[Inorganic Materials, 2005, vol. 41, no. 5, pp. 549-554]

DOI: $10.1134 / \mathrm{S} 0020168506010213$

The editorial staff of Neorganicheskie materialy would like to offer their apologies for the mistakes that crept into the text:

1. The first sentence in the PHENOMENOLOGICAL RESULT VS QUANTUM RESULT section should read as follows:

An essential part of Ginzburg's phenomenological treatment is a phenomenological expansion of the impermeability tensor $\eta_{i j}(\omega, \mathbf{k})$, the inverse of the permittivity tensor $\varepsilon_{i j}(\omega, \mathbf{k})$, in powers of the wavevector $\mathbf{k}, \ldots$

2. Equation (11) should read as follows:

$$
\left|\Omega_{\mathrm{tr}}\right|=\left|\frac{k_{0}^{2} \mu_{\mathrm{tr}} n^{2}}{\omega^{2}-\omega_{\mathrm{lo}}^{2}}\right| \ll 1 .
$$

